\title{
Promoção da saúde como decisão política para a formação do enfermeiro
}

\author{
HEALTH PROMOTION AS A POLITICAL DECISION IN NURSING EDUCATION \\ PROMOCIÓN DE LASALUD COMO DECISIÓN POLÍTICA PARA \\ LA FORMACIÓN DE LOS ENFERMEROS
}

\begin{abstract}
Kenia Lara da Silva', Roseni Rosangela de Sena ${ }^{2}$, Maria Jose Cabral Grillo ${ }^{3}$, Natalia de Cássia Horta ${ }^{4}$, Priscila Malta Coelho Prado ${ }^{5}$
\end{abstract}

1 Enfermeira. Pesquisadora do NUPEPE. Doutoranda em Enfermagem. Professora do Curso de Enfermagem da Pontifícia Universidade Católica de Minas Gerais (PUCMG). Belo

Horizonte, MG, Brasil.

2 Enfermeira. Doutora em Enfermagem. Coordenadora do

Núcleo de Estudos e

Pesquisas sobre

Ensino e Prática de Enfermagem. Professora Emérita da Escola de Enfermagem da Universidade Federal de Minas Gerais (UFMG). Belo Horizonte, MG, Brasil. rosenisena@uol.com.br

3 Enfermeira. Pesquisadora do NUPEPE. Mestre em Enfer-

magem. Professora do Departamento de Enfermagem Básica da Escola de Enfermagem da Universidade Federal de

Minas Gerais (UFMG). Belo Horizonte, MG, Brasil.

4 Enfermeira. Pesquisadora do NUPEPE.

Doutoranda em Enfermagem.

Professora do Curso de Enfermagem da Pontifícia Universidade Católica de Minas Gerais (PUCMG). Belo Horizonte, MG, Brasil.

5 Bolsista do NUPEPE. Graduanda do 8ㅇ Período da Escola de Enfermagem da Universidade Federal de Minas Gerais (UFMG).Belo Horizonte, MG, Brasil.

\section{RESUMO}

Estudo descritivo-exploratório de abordagem qualitativa com o objetivo de analisar a promoção da saúde na formação do enfermeiro. Os cenários foram dois cursos de enfermagem do Estado de Minas Gerais (Brasil). Os resultados indicaram que as duas escolas utilizam espaços de aprendizagem favoráveis à abordagem da promoção da saúde na formação. Ancora-se esta abordagem na necessidade de propiciar aos discentes experiências de aprendizagem com base na imersão na realidade da vida profissional. Na formação, prevalecem tensões entre boas práticas de ensino para a promoção da saúde, reveladas como aquelas que acontecem no cotidiano dos serviços, e práticas que aprisionam os sujeitos em seus modos de pensar e fazer. Concluiu-se que a promoção da saúde é tomada como decisão política para mudança na formação do enfermeiro, embora esta incorporação seja incipiente e heterogênea quanto à formulação teórica, indicando a necessidade de ampliação dos espaços de discussão conceitual e operacional.

\section{DESCRITORES}

Promoção da saúde.

Educação em enfermagem.

Serviços de integração docenteassistencial.

\section{ABSTRACT}

It is a descriptive-exploratory study with a qualitative approach that has the objective of analyzing the health promotion in training nurses. The setting of the research was two nursing undergraduate courses in the State of Minas Gerais, Brazil. The results show that both schools used settings favorable to a health promotion approach in this training. They anchor this approach in the need to provide students experiences of learning from an immersion in the reality of professional life. It is prevalent on formation a tension between good educational pratices for health promotion were revealed those become into the daily of the service and practices that arrest the subjects on their ways of thinking. It is concluded that health promotion is recognized as a political decision in nursing education, although this is yet incipient and has a mixed theoretical formulation, indicating the need to increase opportunities for conceptual and operational discussion.

\section{KEY WORDS}

Health promotion.

Education, nursing.

Teaching care integration services.

\section{RESUMEN}

Este estudio exploratorio descriptivo y cualitativo, analizó la promoción de la salud en la formación de los enfermeros. Los escenarios de investigación fueron dos facultades de Enfermería del Estado de Minas Gerais (Brasil). Los resultados indicaron que las escuelas han practicado aprendizaje favorable al abordaje de la promoción de salud, fundamentada en la necesidad de propiciar experiencias a partir de la inmersión en la vida profesional. Prevalecieron tensiones entre las buenas prácticas de enseñanza para la promoción de la salud, reveladas como las que acontecieron en el cotidiano de los servicios, y las prácticas que aprisionaron a los sujetos en sus modos de pensar e de actuar. Se concluyó que la promoción de la salud fue considerada como decisión política para cambios en la formación del enfermero, a pesar de esa incorporación ser incipiente e heterogénea, fue evidente la necesidad de ampliar los espacios de discusión conceptual y operacional.

\section{DESCRIPTORES}

Promoción de la salud. Educación en enfermería. Servicios de integración docente asistencial. 


\section{INTRODUÇÃO}

$\mathrm{O}$ artigo refere-se ao estudo Abordagem da promoção da saúde na formação do enfermeiro, integrante da pesquisa Promoção da saúde: estratégia política, assistencial, educacional e gerencial para a construção do modelo tecnoassistencial em saúde ${ }^{(\mathrm{a})}$. Buscou-se analisar a abordagem da promoção da saúde na formação do enfermeiro, analisando as práticas de promoção da saúde desenvolvidas durante a formação, identificando os referenciais teórico-conceituais sobre promoção da saúde que sustentam a formação do enfermeiro e as relações existentes entre ensino-serviço-comunidade para viabilizar as práticas de promoção da saúde.

Os debates atuais em torno do tema da educação de profissionais de saúde têm por objetivo a adequação das políticas educacionais, potencializado-as para o estabelecimento de opções mais coerentes com a construção do Sistema Único de Saúde brasileiro e a organização do processo de trabalho no Setor ${ }^{(1-4)}$

A formação dos profissionais de enfermagem no nível da graduação está sendo realizada no país por mais de 500 instituições sob a orientação das Leis de Diretrizes e Bases da Educação Brasileira - $\mathrm{LDB}^{(5)} \mathrm{e}$ das Diretrizes Curriculares Nacionais - DCN- para os cursos da área da saú$\mathrm{de}^{(6)}$. Entretanto, as mudanças nas práticas assistenciais direcionadas à construção da promoção da saúde são ainda incipientes e têm pequena visibilidade no cenário das práticas em saúde justificando, assim, a compreensão desse tema como estratégia de transformação, tanto do ensino quanto da gestão, da concepção e do controle social.

Nesse sentido, é necessário implementar, no ensino, o conceito do processo saúde-doença, adotando-se noções de promoção da saúde em contraposição às práticas direcionadas às dimensões biológica, individual e curativa que caracterizam a formação e a atuação dos profissionais. Agrega-se a necessidade de fomentar as mudanças incorporando conceitos como interdisciplinaridade, intersetorialidade, empoderamento/potencialização de sujeitos e qualidade de vida, entendidos como elementos que sustentam uma nova prática de formação e atuação no campo tendo como referencial a promoção da saúde.

A promoção da saúde configura-se como estratégia de mudança nos modelos tecnoassistenciais, sinalizando a construção de outras possibilidades e a configuração de novos saberes e fazeres que ampliem as alternativas de qualidade de saúde e vida da população, de intervenção junto

(a) Pesquisa financiada pela Fundação de Amparo à Pesquisa do Estado de Minas Gerais (FAPEMIG). Processo: 11560 - FAPEMIG/EENF/EMI/SUS TECNOASSISTENCIAL (AP). aos sujeitos e da compreensão do processo saúde-doença como produção social ${ }^{(7-8)}$. Apesar do grande avanço em sua formulação, a concepção de promoção da saúde não é devidamente incorporada aos projetos politico-pedagógicos dos cursos da área de saúde nem às práticas educativas, realizadas junto à população.

Com esta abordagem da promoção da saúde na formação dos enfermeiros, espera-se contribuir com a reflexão sobre o tema e com a identificação de necessidades de mudanças no ensino de enfermagem que superem modelos hegemônicos de educação, de gestão e de atenção à saúde. Pretende-se também contribuir para a construção ou reconstrução de referenciais teóricos e metodológicos que alicercem uma prática pedagógica e assistencial visando à promoção da saúde.

\section{MÉTODO}

O estudo caracteriza-se como descritivo-exploratório com abordagem qualitativa, orientado pela concepção teóricofilosófica da dialética. As questões éticas foram observadas em todas as etapas da pesquisa, atendendo a Resolução 196/96 do Conselho Nacional de Ética (Parecer n. 463/06).

Os cenários da pesquisa foram dois cursos de graduação em enfermagem do Estado de Minas Gerais (Brasil), uma instituição privada e uma pública.

Numa primeira fase, foram entrevistadas as coordenadoras dos dois cursos, utilizando-se um roteiro semi-estruturado. No momento da entrevista, foi solicitada às coordenadoras a descrição das experiências exitosas para a promoção da saúde vivenciadas na formação do enfermeiro naquela instituição. A segunda etapa da coleta de dados consistiu na verificação in loco de situações marcadoras indicadas, resultando em entrevistas com 2 docentes, 5 estudantes e 11 profissionais dos serviços de saúde que recebem os estudantes de enfermagem.

Para a análise dos dados empíricos, utilizou-se a técnica de análise de discurso. Foram realizadas leituras exaustivas buscando-se apreender as idéias centrais, ou seja, estruturas de significados apresentadas nas falas dos participantes. Do agrupamento das idéias centrais, originou-se uma rede de significados expressas pelos participantes do estudo que se relacionavam com as políticas e as ações pedagógicas que favorecem a incorporação de concepções e práticas de promoção da saúde na formação do enfermeiro. A rede de significados foi analisada de acordo com os objetivos do estudo e foram escolhidos dois analisadores para a construção deste trabalho: Diversificação dos cenários de aprendizagem e a promoção da saúde e Práticas aprisionadas X boas práticas de ensino para a promoção da saúde. 


\section{RESULTADOS E DISCUSSÃO}

As duas escolas, cenários do estudo, apresentam estruturas político-administrativas distintas, o que as diferencia no funcionamento, na composição da força de trabalho docente, na concepção que orienta o projeto político-pedagógico e nas práticas pedagógicas e de inserção nos cenários de aprendizagem. Contudo, ambas fazem referência em seu projeto político-pedagógico às discussões nacionais sobre a formação do enfermeiro, em especial as Diretrizes Curriculares e aquelas relacionadas à abordagem da promoção da saúde enquanto política pública.

As coordenadoras afirmam que a formação do enfermeiro tem direcionado esforços no sentido de construir modelos de ensino que ofereçam ferramentas e desenvolvam as competências para a atuação profissional, reconhecendo e contribuindo para a construção de novas práticas em saúde.

Considerar esses aspectos na formação do enfermeiro permite inferir que o projeto ético-político da enfermagem deve produzir conhecimentos e prática social que superem a reprodução de práticas hegemônicas e dominantes no campo da saúde, num verdadeiro processo de mudança da formação ${ }^{(2-3)}$. Nos discursos das coordenadoras é revelada a compreensão de que o processo de mudança na formação do enfermeiro é gradual e desafiante por requerer ações estratégicas no sentido de incorporar a promoção da saúde como referencial no ensino e nas práticas sanitárias, e implicação de todos os atores do processo ensino-aprendizagem.

A verificação in loco das experiências indicadas como marcadoras da aprendizagem para promoção da saúde permitiu analisar o referencial de promoção da saúde que orienta a formação e a relação ensino-serviço-comunidade subjacente à sustentabilidade desse conceito.

Os discursos apontam que no processo de formação há espaços para que docentes e estudantes desenvolvam práticas de promoção da saúde. Em um dos discursos, faz-se uma reflexão sobre o conceito de promoção apontando a imprecisão conceitual que permeia na formação e na atenção as práticas denominadas de promoção da saúde ${ }^{(9)}$. Revela ainda o distanciamento entre a formulação conceitual e a inovação e concretude das práticas de promoção nos cenários de aprendizagem.

Um dos aspectos importantes analisados nos discursos refere-se à diversificação dos cenários de aprendizagem e à potencialidade que essa construção apresenta para a mudança no ensino. Os cenários de aprendizagem permitem a ampliação do contato entre estudantes, profissionais do serviço e comunidade e, ao ampliar essa superfície, permitem a incorporação de novos conceitos como a abordagem sócio-política e de qualidade de vida, empoderamento e ampliação da capacidade das comunidades nas decisões sobre a saúde, além do indispensável estabelecimento das parcerias intersetoriais na construção de políticas públicas que incidem sobre a saúde.
Os estudantes dos dois cenários ressaltam a importância do espaço do trabalho como lócus privilegiado de relações com os serviços de saúde e com a comunidade o que constitui um cenário de expressão do espírito criativo e favorável à aprendizagem. Nesse sentido, salientam as experiências indicadas como marcadoras de aprendizagem de promoção da saúde como oportunidade de confrontar a realidade com o aprendido.

A análise das boas práticas indicadas nos cenários do estudo revela a utilização de metodologias ativas de ensino que permitem o protagonismo dos estudantes e a construção de relações de vínculos com os serviços e com a população. As relações ensino-serviço-comunidade para viabilizar as práticas de promoção da saúde foram expressas nos discursos tendo como referência os avanços nos dois cursos e os desafios a serem enfrentados para as mudanças estruturanntes dos modelos de formação das duas instituições cenários do estudo.

Os profissionais de serviço reconhecem o papel do estudante na renovação do conhecimento e conseqüentemente nas práticas dos serviços, favorecendo a reorganização com novas propostas que potencializam as ações de promoção da saúde.

O confronto entre as concepções expressas pelos estudantes e pelos profissionais dos serviços de saúde leva a questionar a potência da presença dos estudantes na mudança das práticas em saúde, condição afirmada por um dos profissionais como principal motivação para a continuidade do estágio (a presença dos estudantes provoca uma crise existencial nos serviços). Entretanto, não se observam, na formação do enfermeiro, mecanismos e estratégias de ensino-aprendizagem que incluam a mudança de concepção sobre os referenciais que sustentam a promoção da saúde e sua materialidade na transformação das práticas assistenciais em saúde.

Infere-se que essa contradição remete a dois determinantes inter-relacionados:

$1^{\circ}$ ) A cristalização de práticas assistenciais em saúde que reiteram um modelo no qual as ações de saúde são realizadas apenas no sentido de agir sobre os efeitos do adoecer, não incorporando, ainda, práticas nos espaços fora dos serviços de saúde, transcendendo a responsabilidade de proporcionar ações de recuperação da saúde com enfoque curativo;

$2^{\circ}$ ) A presença dos estudantes nos cenários é capaz de mobilizar novas práticas assistenciais e de cuidado, com foco na promoção da saúde mas são poucos os mecanismos criados pelas instituições de ensino para garantir a sustentabilidade das mudanças provocadas nos serviços. Assim, mesmo que potentes para imprimir uma nova lógica no modelo tecnoassistencial em saúde, as rupturas e as soluções de continuidade no processo enfraquecem a capacidade das iniciativas disparadas. 
De modo geral, os sujeitos implicados no novo modo de agir são os discentes, numa relação construída entre discentes, profissionais e comunidade. Os docentes ainda estão arraigados em metodologias diretivas que não permitem captar a dinâmica das relações no mundo do trabalho. Quando se toma como foco a promoção da saúde, a tensão entre as boas práticas e as práticas que aprisionam os sujeitos e sua capacidade criativa fica mais evidente no ambiente acadêmico. Os discursos dos professores não sinalizam a riqueza das práticas vivenciadas no cotidiano das relações no serviço. Assim, em conseqüência do processo de trabalho dos professores que não permitem a aderência à superfície de contato presente nos cenários de aprendizagem, o professor não entra nos acontecimentos da promoção da saúde, embora não sejam estranhos a ele. Contribui para esse distanciamento e para a dificuldade de incorporação das boas práticas no cotidiano do ensino o arranjo dos projetos pedagógicos presos a certos modos de fazer e de pensar. Amplia-se a dissociação entre teoria e prática que prevalece na formação.

A promoção da saúde está colocada como concepção que revela uma decisão política de mudança na formação do enfermeiro, mas, como a organização de currículo é por conteúdo, a incorporação desse conceito - que não se traduz na visão linear e classificatória do setor saúde na perspectiva tradicional do processo saúde-doença - fica prejudicada.

É reconhecido que os princípios da educação em enfermagem desenvolvidos nas duas instituições exigem mudanças nas práticas de ensino superando o modelo biologicista e a natureza setorial que caracterizam a formação e a atuação dos profissionais de saúde para ser assumida como um ideário do coletivo das escolas. Entretanto, carecem de maior valorização da integração discente-serviço-comunidade como condição para dar sustentabilidade ao processo de mudança na formação do enfermeiro, contextualizando o educando na realidade do serviço e da comunidade na qual está inserido e nas práticas assistenciais.

\section{REFERÊNCIAS}

1. Feuerwerker LCM. Além do discurso da mudança na educação médica: processos e resultados. São Paulo: Hucitec; 2002.

2. Feuerwerker LCM. A construção de sujeitos no processo de mudança na formação dos profissionais de saúde. Divulg Saúde Debate. 2000;(22):18-24

3. Silva KL, Sena RR. Nursing education: seeking critical-reflexive education and professional competencies. Rev Lat Am Enferm. 2006;14(5):755-61.

4. Silva KL, Sena RR. A formação do enfermeiro: construindo a integralidade do cuidado. Rev Bras Enferm. 2006;59(4):488-91.

5. Brasil. Lei n. 9394, de 20 de dezembro de 1996. Estabelece as Diretrizes e bases da Educação Nacional. Diário Oficial da União, Brasília, 23 dez. 1996. Seção 1, p. 27833-41.
Assim, a construção de cenários favoráveis a novas práticas deve sustentar-se e orientar-se pela participação dos sujeitos na elaboração de propostas de mudanças nos modelos de ensino e no estabelecimento de instâncias tripartites para a discussão e deliberação sobre a análise da situação atual e projeção de mudanças ${ }^{(10)}$. Defende-se essa abordagem na necessidade de construção de um sistema de saúde que tem como pressuposto a concepção ampliada de saúde e que se compromete com a regulação da formação dos profissionais para o setor.

\section{CONSIDERAÇÕES FINAIS}

Conclui-se que a promoção da saúde é tomada como decisão política para mudança na formação do enfermeiro, apesar desta incorporação ser incipiente e heterogênea quanto à formulação teórica, indicando a necessidade de ampliação dos espaços de discussão conceitual e ope-racional. A formação de profissionais centrados na realidade dos serviços de saúde e que atendam às demandas de boas práticas de promoção da saúde exige que universidade e serviços estejam juntos nas discussões do processo de formação.

Enfatiza-se a necessidade de implementar no ensino o conceito ampliado do processo saúde-doença, adotandose noções de promoção da saúde em contraposição à natureza setorial que caracteriza a formação e a atuação dos profissionais, considerando-se o enfoque social, comunitário e político como determinante das respostas efetivas em saúde. Portanto, para promover saúde, é preciso construir políticas públicas intersetoriais voltadas para a melhoria da qualidade de vida, eqüidade na produção e no consumo de ações e serviços de saúde, inclusão social e afirmação da cidadania.

6. Brasil. Ministério da Educação e Cultura. Conselho Nacional de Educação Brasil. Câmara de Educação Superior. Resolução CNE/ CES n. 3, de 07 de novembro de 2001. Diretrizes Curriculares Nacionais do Curso de Graduação em Enfermagem. Diário Oficial da União, Brasília, 9 nov. 2001. Seção 1, p. 37.

7. Brasil. Ministério da Saúde. Política Nacional de Promoção da Saúde. Brasília; 2006.

8. Buss PM. Promoção da saúde e qualidade de vida. Ciênc Saúde Coletiva. 2000;5(1):167-77.

9. Czeresnia D. O conceito de saúde e a diferença entre prevenção e promoção. In: Czeresnia D, Freitas CM, organizadoras. Promoção da saúde: conceitos, reflexões, tendências. Rio de Janeiro: FIOCRUZ; 2003. p. 39-54.

10. Ceccim RB, Feuerwerker LCM. O quadrilátero da formação para a área da saúde: ensino, gestão, atenção e controle social. PHISIS Rev Saúde Coletiva. 2004;14(1):41-65. 